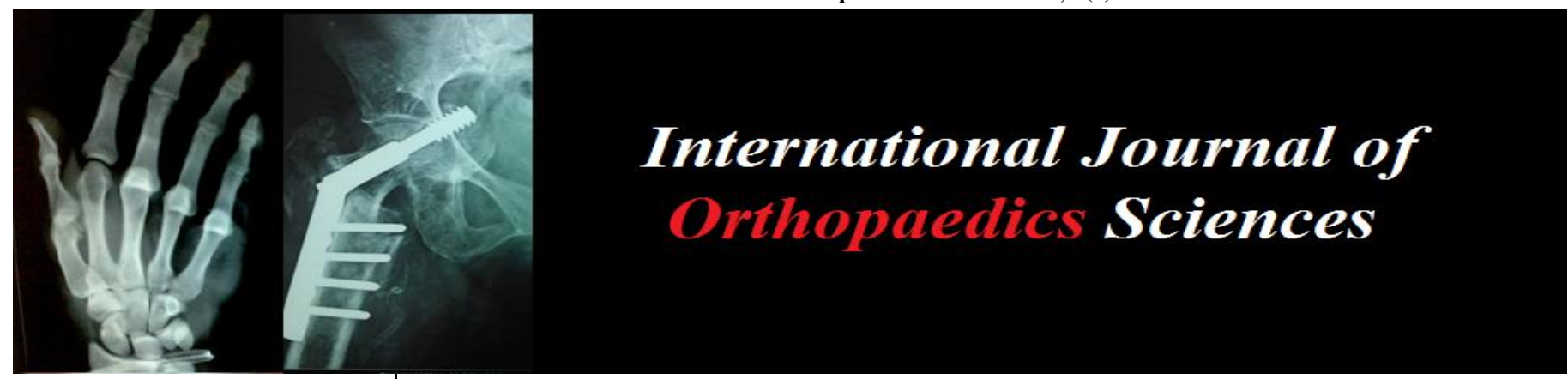

E-ISSN: 2395-1958

P-ISSN: 2706-6630

IJOS 2021; 7(3): 116-124

(C) 2021 IJOS

www.orthopaper.com

Received: 16-04-2021

Accepted: 19-06-2021

Dr Bula Ratna Kumar

S Resident, Department of

Orthopaedics, Vydehi Institute of Medical Sciences and Research

Centre, Bengaluru, Karnataka, India

Dr. Aravind JD

Professor and Unit Chief,

Department of Orthopaedics,

Vydehi Institute of Medical

Sciences and Research Centre,

Bengaluru, Karnataka, India

Dr. Hiranya Kumar S

Professor and HOD, Department of Orthopaedics, Vydehi

Institute of Medical Sciences and

Research Centre, Bengaluru,

Karnataka, India
Corresponding Author:

Dr Bula Ratna Kumar

S Resident, Department of

Orthopaedics, Vydehi Institute

of Medical Sciences and Research

Centre, Bengaluru, Karnataka,

India

\section{A comparative study of clinical and functional outcome of arthroscopic anterior cruciate ligament reconstruction using hamstring graft with aperture fixation versus suspensory device fixation}

\author{
Dr. Bula Ratna Kumar, Dr. Aravind JD and Dr. Hiranya Kumar S
}

DOI: https://doi.org/10.22271/ortho.2021.v7.i3b.2737

\section{Abstract}

Introduction: Anterior cruciate ligament injury is a common injury, frequently occurs in sport. Anatomic ACL reconstruction with autograft or allografts remains a technically demanding procedure. Fixation of replacement graft can be classified into direct and indirect methods. Graft fixation continues to be a weak link in the early rehabilitation process. Our study aimed to compare clinical and functional results between aperture fixation and suspensory fixation of hamstring graft on femoral side.

Methods: It is a prospective cohort study conducted on 30 patients, 15 patients in each group with at least 1 year follow up, who underwent ACL reconstruction with autogenous hamstring graft at Vydehi Institute of Medical Sciences and Research Centre, Bangalore from January 2018 to May 2019. Patients aged between 20-46 years of both genders were included in study. The aperture fixation group underwent interference screw fixation at both femoral and tibial tunnels. The suspensory fixation group underwent endobutton fixation on the femoral side and interference screw on tibial side. Patients in both the groups were examined prior to surgery and regularly till 12 months post-surgery. They were compared for clinical and functional outcome with Tegner Lysholm knee score.

Results: There was statistically significant improvement in Tegner Lysholm knee score from preoperative to 12 months post-operative with $\mathrm{p}$ value $<0.001$ in both groups, whereas there was no statistically significance difference in post-operative scores at the end of 1 year between the two groups. Conclusion: The clinical and functional results in our study clarified that both techniques are effective and safe methods for femoral hamstring fixation in ACL-reconstruction. However, further studies are needed to confirm these findings during a longer follow up.

Keywords: Anterior cruciate ligament reconstruction, arthroscopy, graft fixation, lysholm knee score

\section{Introduction}

Anatomic ACL reconstruction with autograft or allografts remains a technically demanding procedure. Fixation of replacement graft can be classified into direct and indirect methods. Direct fixation devices include interference screws, staples, washers and cross pins. Indirect fixation devices include polyester tape/titanium button and suture-post ${ }^{[1]}$. The best fixation method for the soft tissue hamstring graft is still being debated by orthopaedic surgeons ${ }^{[2]}$.

Graft fixation continues to be a weak link in the early rehabilitation process ${ }^{[3]}$. A major cause for concern with the use of hamstring autografts is the soft tissue can take up to 12 weeks to heal to the osseous tunnel ${ }^{[4,5]}$. Thus, a secure fixation technique is needed to withstand the forces on the graft resulting from current rehabilitation protocols that allow for unrestricted ROM, weightbearing, and early return to athletic activity after ACL reconstruction ${ }^{[4,6]}$.

According to a study by Kim et al, the type of graft or fixation device did not make significant differences in clinical outcomes or stability of ACL reconstruction ${ }^{[7]}$.

Multiple studies have found no significant difference between aperture fixation and suspensory fixation ${ }^{[8-12]}$.

A meta-analysis study by Browning et al, concluded improved overall arthrometric stability and fewer graft ruptures with suspensory fixation compared to aperture fixation ${ }^{[2]}$. 
The purpose of present study is to compare clinical and functional outcomes between aperture and suspensory fixation of a quadrupled hamstring tendon autograft on femoral side in ACL reconstruction.

\section{Materials and Methods}

It is a prospective cohort study conducted among patients undergoing arthroscopic anterior cruciate ligament reconstruction in the department of orthopaedics at Vydehi institute of medical sciences and research centre, Bengaluru during January 2018 to May 2019. Ethics clearance was obtained prior to recruitment of the patients for study. Patients were included in study after obtaining informed consent. Patients aged between 20-46 years of both genders were included in the study.

In our study 72 patients were initially considered eligible to be included in the study. 42 patients were eventually excluded from the study due to no follow up data. Two groups with 15 patients each, with at least 1 year follow up were included in the study. The aperture fixation group underwent interference screw fixation at both femoral and tibial tunnels. The suspensory fixation group underwent endobutton fixation on the femoral side and interference screw on tibial side.

All patients with exclusive ACL tear clinically and radiologically with or without associated menisci injuries which do not alter postoperative rehabilitation were included in study. Patients with multi ligament injuries, meniscal injuries requiring repair, previous knee surgeries, associated osteoarthritis and avulsion fractures were excluded from study.

All the patients in both the groups, interference screw (group 1) \& endobutton (group 2) evaluate pre operatively for laxity, clinically with Lachman, Anterior drawer and Pivot shift test and assessed functionally with Tegner Lysholm knee scoring system which includes 1.limp 2.support 3.locking 4.instability 5.pain 6.swelling 7.climbing stairs 8.squatting and grading will be done as poor, fair, good or excellent based on total score. All patients were also evaluated by Xray and MRI of knee preoperatively. Operations were done by different surgeons using standard portals following diagnostic arthroscopy, ACL reconstruction with autogenous quadrupled hamstring graft. Intraoperative (tourniquet time, graft rupture) and post-operative (persistent anterior knee pain, persistent effusion, infection, DVT/PE) parameters were compared in between the groups. All patients were subjected same rehabilitation protocol post operatively. Patients were regularly followed till 12 months, laxity tests and functional outcome with Lysholm score were assessed at 2 weeks, 3, 6 and 12 months.

\section{Statistical Analysis}

Collected Data was analyzed using IBM, SPSS version 21. All quantitative variables were expressed as Mean +/- SD. All qualitative (categorical) variable were expressed as percentages or frequencies. To find statistical significance between Intergroup Paired $\mathrm{T}$ Test and in-between both groups comparison was done using Unpaired $\mathrm{T}$ Test. $\mathrm{P}$ value less than 0.01 was considered as statistically significant.

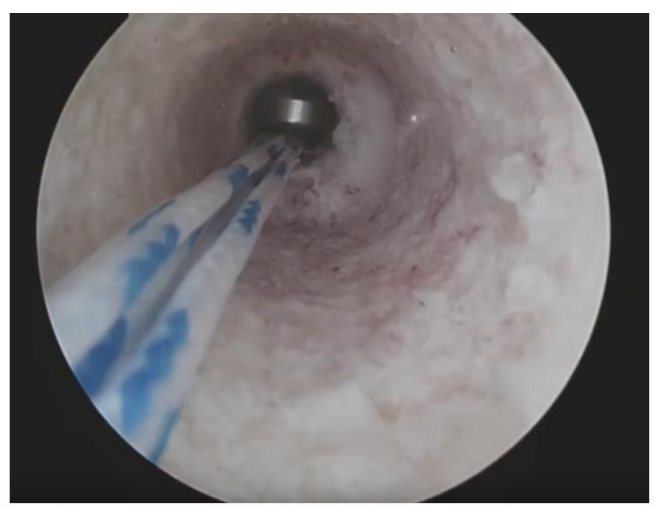

b

Fig 1: Graft fixation femoral side using (a) interference screw (b) endobutton on femoral side

\section{Results}

Majority of the patients were of third decade of life, with youngest patient being 20 years (in both the groups) and the oldest being 46years (Endobutton), with mean age of $27.40 \pm$ 5.46years in Group 1(Interference screw) and $30.53 \pm$ 7.56years in Group 2 (Endobutton).
In our study male preponderance was noted, similar to other studies with $90 \%$ males and $10 \%$ female in overall study population. $90 \%$ patients presented within 2 years of injury, 19 patients $(63.3 \%)$ presented between 6 to 12 months, 8 patients $(26.6 \%)$ presented between 13 to 24 months, and 3 patients $(10 \%)$ presented between 2 to 5 years.

Table 1: Age Distribution

\begin{tabular}{|c|l|l|l|l|}
\hline AGE IN YEARS & GROUP 1 (INTERFERENCE SCREW) & \multicolumn{2}{|c|}{ GROUP 2 (ENDOBUTTON) } \\
\hline & \multicolumn{1}{|c|}{ NUMBER } & PERCENTAGE & NUMBER & PERCENTAGE \\
\hline$\leq 20$ & 2 & 13.3 & 1 & 6.6 \\
\hline $21-30$ & 8 & 53.4 & 10 & 66.8 \\
\hline $31-40$ & 5 & 33.3 & 2 & 13.3 \\
\hline $41-50$ & 0 & 0 & 2 & 13.3 \\
\hline TOTAL & 15 & 100 & 15 & 100 \\
\hline
\end{tabular}


Nature of the injury in our study was mainly, Sports injury, which accounts for 16 patients (53.4\%) and rest were Road traffic accident, Injury at work and slip and fall which account for $7(23.3 \%), 6(20 \%)$ and $1(3.3 \%)$ respectively.

Right side was affected in $19(63.3 \%)$ patients and left side 11 (36.6\%) patients.

In the study population, 16 patients had isolated ACL injury and remaining 14 patients had ACL associated meniscal injuries (40\% of Group 1 and $53.3 \%$ of Group 2). Of the 6 patients in Group 1, 4 patients had medical meniscal tear and remaining 2 had lateral meniscal tear. And among 8 patients in Group 2, 3 patients had medical meniscal injury, 3 had both medial and lateral meniscal injury and remaining 2 had isolated lateral meniscal injury. But none required any intervention for meniscal injury.

Lachman's test was positive preoperatively in $80 \%$ in Group 1 (Interference screw) and $86.7 \%$ in Group 2 (Endobutton), with $100 \%$ negative at the end of 12 months in both groups. Anterior drawer test was positive in $80 \%$ in Group 1 and $73.3 \%$ in Group 2, with $100 \%$ negative at the end of 12

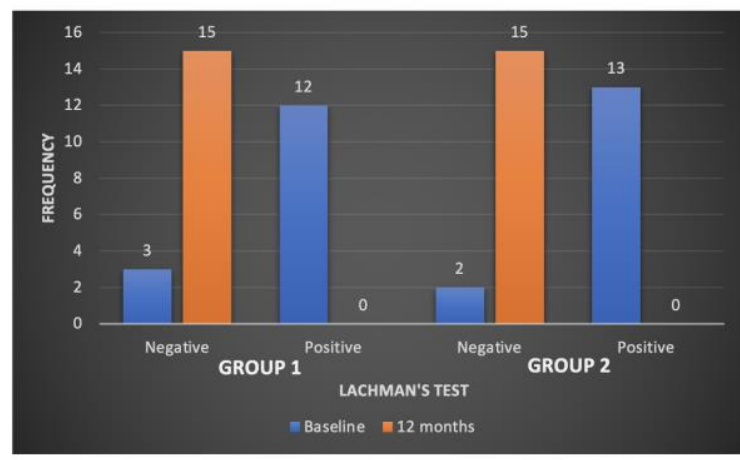

\section{A}

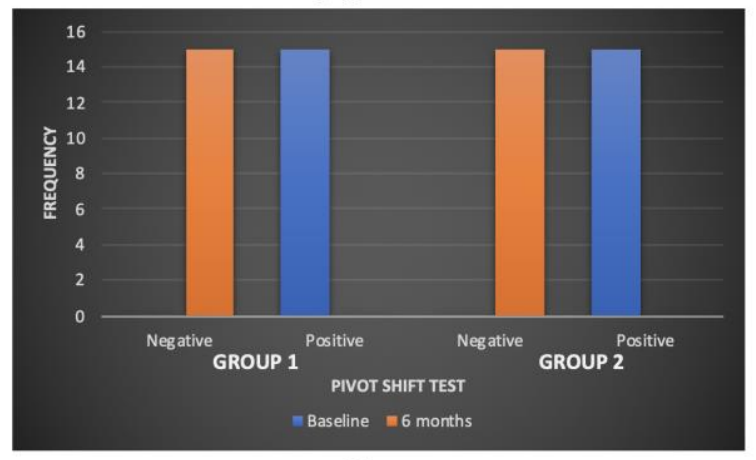

$\mathrm{C}$ months in both groups. Pivot shift test was positive in $100 \%$ in Group 1 and $100 \%$ in Group 2 when examined under anaesthesia preoperatively, with $100 \%$ negative at the end of 12 months in both groups. The pivot shift test was deferred till 6 months post operatively.

Among 15 patients in Group 1, 11 (73.3\%) patients had no donor site pathology, 1(6.7\%) had tenderness at the Graft harvest site, 2(13.3) patients had numbness in the harvest site and lateral to the harvest site, 1(6.7\%) patient had a hypertrophic scar. Out of the 15 patients in Group 2, 11 (73.3\%) patients had no donor site pathology, 2(13.3\%) had tenderness at the Graft harvest site, 2(13.4) patients had numbness in the harvest site and lateral to the harvest site.

In Group 1, 2 patients had joint effusion, patellar tap was positive and in Group 2, 1 patient had effusion, patellar tap was positive. The effusion gradually reduced in all the patients without requiring any intervention. There were no graft ruptures, persistent anterior knee pain, infection or Deep vein thrombosis/Pulmonary Embolism.

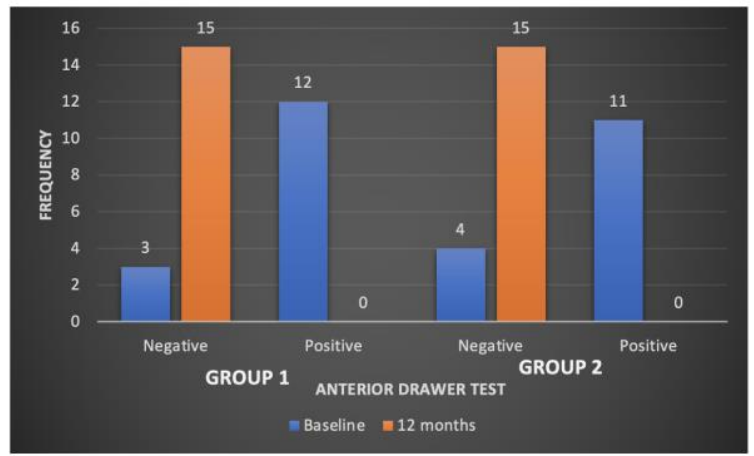

B

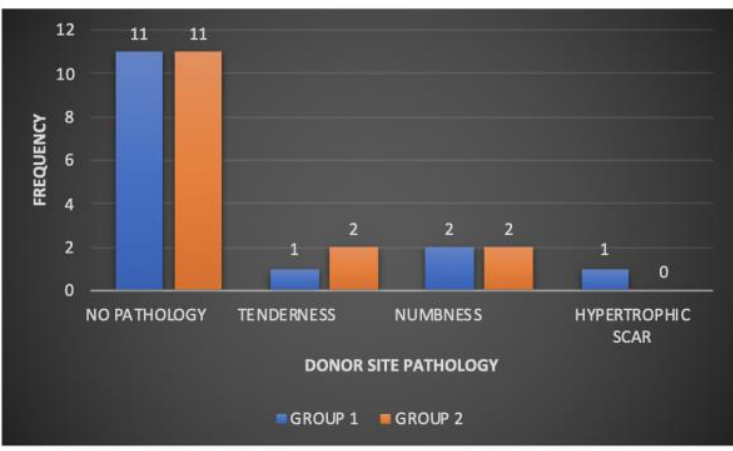

$\mathrm{D}$

Fig 2: A-Lachman test, B-Anterior Drawer test, C-Pivot shift test - preoperative and postoperative in both groups, D-Donor site pathology

The preoperative Tegner Lysholm knee score was poor in all the patients among both the groups $(<65)$. Post operatively in Group 1, 12 patients (80\%) had excellent functional outcome while 3 patients (20\%) had good outcome. In Group 2 (Endobutton), 11 patients $(73 \%)$ had excellent functional outcome while 4 patients $(27 \%)$ had good outcome.
There was statistically significant improvement in Tegner Lysholm knee score from preoperative to postoperative with $\mathrm{p}$ value $<0.001$ in both groups, whereas there was no statistically significance difference in post-operative scores between the two groups. 


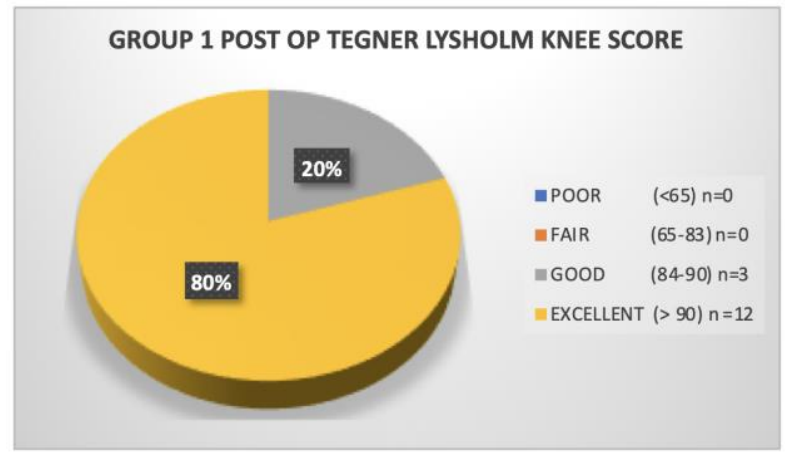

A

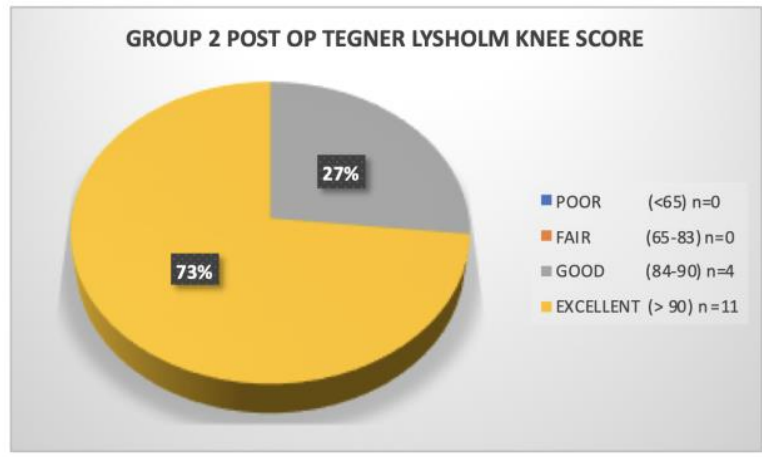

B

Fig 3: Post-op (12 months) Tegner Lysholm knee score Grade in (A) Group 1 (B)Group 2

Table 2: A: Association between pre op and post op Tegner Lysholm knee score in Group 1 B: Association between pre op and post op Tegner Lysholm knee score in Group 2 C: Association between post op Tegner Lysholm knee score between Group 1 \& Group 2

\begin{tabular}{|l|c|c|c|c|}
\hline Tegner Lysholm knee score & N & Mean & Std. Deviation & P Value \\
\hline Pre - op & 15 & 43.47 & 10.120 & \multirow{2}{*}{0.000} \\
\hline At 12 months & 15 & 96.07 & 5.625 & \\
\hline
\end{tabular}

\begin{tabular}{|l|c|c|c|c|}
\hline \multicolumn{1}{|c|}{ Tegner Lysholm knee score } & N & Mean & Std. Deviation & P Value \\
\hline Pre - op & 15 & 41.33 & 12.146 & \multirow{2}{*}{0.000} \\
\hline At 12 months & 15 & 95.40 & 5.667 & \\
\hline
\end{tabular}

\begin{tabular}{|l|c|c|c|c|}
\hline Tegner Lysholm knee score & N & Mean & Std. Deviation & P Value \\
\hline Group 1 & 15 & 96.07 & 5.625 & \multirow{2}{*}{0.749} \\
\hline Group 2 & 15 & 95.40 & 5.667 & \\
\hline
\end{tabular}

\section{Discussion}

The aim of ACL reconstruction procedure is to allow the patient to return to normal daily activity, avoiding further meniscal damage and prevent osteoarthritis and having normal knee function. Several factors are identified as significant influence for the biomechanical characteristics and functional outcome of an ACL reconstructed knee joint. These factors are ${ }^{[13]}$ :

1. Individual choice of graft material using either bone patellar tendon, hamstring graft, quadriceps tendon, various synthetic grafts or allograft.

2. Anatomical tunnel placement within the footprints of native ACL

3. Adequate substitute tension after preconditioning graft by cycling

4. Anatomical graft fixation.

The most commonly used grafts in ACL reconstruction are bone patellar tendon and hamstring graft. Each has its merits and demerits, in our study we used hamstring graft for ACL reconstruction in all study population.

Irrespective of the type of graft used, fixation of the graft is usually the site of failure rather than the graft itself. This usually occurs during early rehabilitation phase as the graft integration has not occurred during this time. The fixation is of less significance after two to three months as the graft would have integrated with the bone ${ }^{[14-17]}$. There are chiefly two types of fixation devices used in ACL reconstruction in bone tunnels: Aperture fixation and Suspensory fixation. However, debate continues about which technique is better.

The purpose of our study was to compare postoperative knee stability, graft failure, and clinical and functional outcomes between aperture (Interference screw) and suspensory fixation (endobutton) of a hamstring tendon autograft on femoral bone tunnel in ACL reconstruction.

Most of the patients in this study were from third decade of life. Mean age of the patient in Group 1(Interference screw) is 27.40 years and Group 2 (Endobutton) is 30.53years. The combined mean is about 28.9 years. This indicates that young active population were most often involved. In study by Jomha NM ${ }^{[18]}$, mean age was of 26 years, in D Choudhary et al. ${ }^{[19]}$ had 27years as mean age, Williams RJ et al. ${ }^{[20]}$ was 33 years, Mahir et al. ${ }^{[21]}$ was 24years and Kumar PA ${ }^{[22]}$ was 27years. Albert $\mathrm{O}$ Gee in his study concluded that ACL reconstruction when done on patients aged > 40 years produced equivalent functional outcomes when compared with patients aged $\leq 25$ years, without at an increase complication rate at an average of $>5$ years after surgery ${ }^{[23]}$. Majority of the patients in our study were males (90\%) i.e., 27 out of 30 . There were 3 female's patients, 1patient in Group 1 (Interference screw) and 2 patients in Group 2 (Endobutton). In comparison to Jomha NM ${ }^{[18]}$, D Choudhary et al. ${ }^{[19]}$, Williams RJ et al. [20], Mahir et al ${ }^{[21]}$ and Kumar PA [22] where $73 \%, 93 \%, 59 \%, 100 \%$ and $97.1 \%$ was the percentage 
of males in their study respectively.

However, in study conducted by Wild $\mathrm{CY}^{24}$ has stated that girls involving in sports activity have 8 times more chance of suffering ACL injury than boys. The reason given was:

- Narrower intercondylar notch and smaller ACL

- Wider pelvis

- More lax ligaments

- Slower reflex time

- Greater quadriceps/hamstring strength ratio

- Changes in Estrogen level

- Flat footed landing

However, in comparing the outcome between male and females after ACL reconstruction, Ferrari JD [25], has stated that outcomes are similar in both groups with equal chances of failure.

Mode of injury to cause the ACL tear in the study were Sports injury which accounts for $16(53.4 \%)$ of total injury, whereas Road traffic accident and Injury at work accounted for $7(23.3 \%)$ and $6(20 \%)$ of total injury respectively. And 1 patient $(3.3 \%)$ had a slip and fall. Chappell JD et al. ${ }^{[26]}$ suggested that ACL torn during sports activity are usually from non-contact sports. RTA is due to direct trauma to the knee or may be due to dashboard injury or fall from height with the twisting force.

Associated meniscus tear was present in $6(40 \%)$ and 8 $(53.3 \%)$ patients of Group 1 and Group 2 respectively. Of the 6 patients in Group 1, 4 patients had medical meniscal tear and remaining 2 had lateral meniscal tear. And of the 8 patients in Group 2, 3 patients had medical meniscal injury, 3 had both medial and lateral meniscal injury and remaining 2 had isolated lateral meniscal injury. In study by D Choudhary et $a l^{19}$ had Medial meniscal tear in $37.9 \%$ and lateral meniscal tear $16.7 \%$ of study population. In a study conducted by Hagino T et al. ${ }^{[27]}$, the incidence of meniscal tear associated with injury is higher in chronic case. Early ACL reconstruction is suggested to prevent secondary meniscal tear.

Minimum time taken for surgery following injury in the study subjects was 6 months and maximum was 48 months. Majority of the patients $(90 \%)$ presented within 2 years of injury. The average time taken for surgery in Group 1 is 16.60 months and average time taken for surgery in Group 2 is 13.40 months. The mean average of both the groups were 15.0 months. In the study by Jomha NM ${ }^{[18]}, 35.6 \%$ were operated within 3 weeks, $18.6 \%$ between 3 to 12 weeks and $45.8 \%$ after 12 weeks from the time of injury. In the study conducted by Nikolaos K Paschos ${ }^{[28]}$, the ACL injury is a significant factor for developing secondary knee osteoarthritis. The risk of osteoarthritis is doubled each year following ACL injury. Usually the ACL reconstruction is delayed until 6 to 8 weeks after injury. Shelbourne et al. ${ }^{[29,30]}$ in their study concluded that ACL reconstruction within first week of injury had a high incidence of arthrofibrosis compared to those who underwent surgery after 3 weeks. An interval of 6 to 8 weeks is necessary between injury and surgery for the injured knee to become free of irritation (swelling, effusion, erythema).

The common complications encountered in Arthroscopic ACL reconstruction are ${ }^{[31]}$ :

- Persistent anterior knee pain

- Instability

- Joint swelling (Effusion)

- Infection
- Stiff knee

- Deep venous thrombosis

In our study 2 patients (13.3\%) from Group 1 and 1 patient (6.6\%) from Group 2 had mild knee effusion, which over a period, gradually reduced. Tenderness was present at the donor graft site in 1 patient $(6.6 \%)$ and 2 patients $(13.3 \%)$ in group 1 and group 2 respectively. Numbness was present at the donor graft site in 2 patients $(13.3 \%)$ in both group 1 and group 2. Hypertrophic scar was present in 1 patient at the donor graft site in group 1. There were no graft ruptures, persistent anterior knee pain, infection or Deep vein thrombosis/Pulmonary Embolism.

Tunnel widening after ACL reconstruction is multifactorial, with several biological and mechanical factors. Among them are graft tunnel motion, stress deprivation of the bone within the tunnel wall, improper graft tunnel placement, tunnel positioning, graft fixation method, aggressive rehabilitation and bone quality. Sagittal graft motion known as the windshield wiper effect can also occur leading to tunnel widening. CT scan is helpful to measure tunnel widening post operatively ${ }^{[32-36]}$.

The mean tourniquet time for group 1 and group 2 was 103.3 and 106.6 minutes respectively.

In the post-operative clinical outcome of both the groups is measured by Ligament examination which includes Lachman, Anterior Drawer and Pivot shift test at the end of 6 and 12 months for AP translation. And functional outcome was measured by Tegner Lysholm knee score, in this scoring we take various factors including limp, support, locking, instability, pain, swelling, climbing stairs and squatting. Based on the total score, grading was done as poor, fair, good or excellent. Anterior stability tests were negative in all 30 patients at the end of 6 and 12 months. There was significant improvement in functional outcome based on Tegner Lysholm knee score from pre-operative to post-operative in both the groups. But there was no statistically significant difference in post-operative scores between the two groups. Functional outcomes are comparable between both the groups with mean Tegner Lysholm knee score of group 1 and group 2 are 96.07 and 95.40 respectively.

The results were comparable with studies by Jomha ${ }^{[18]}$, D Choudhary et al [19], Williams RJ et al. ${ }^{[20]}$, Mahir et al. [21] and Kumar PA ${ }^{[22]}$ who had average Tegner Lysholm knee score of 94, 92, 91,93.5 and 90 respectively.

Meta-analysis study by Browning WM et al in USA, from 2004-2014 on single bundle ACL reconstructions using 4 stranded hamstring autograft in which 21 studies utilized aperture fixation and 20 utilized suspensory fixation with minimum 24 months follow up, stability and clinical outcome were compared between aperture and suspensory fixation using Lachman or pivot shift test or KT-1000 side-to-side difference (for stability) and clinical outcomes with International Knee Documentation Committee (IKDC), Tegner and Lysholm scores. The study concluded improved overall arthrometric stability and fewer graft ruptures with suspensory fixation compared to aperture fixation and no difference in IKDC, Lysholm, Lachman and pivot-shift ${ }^{[2]}$.

Meta-analysis study by Colvin Aet al in USA in 2010. Study was performed on eight studies (those met the criteria among 1058 initial studies) reporting on surgical failures and postoperative International Knee Documentation Committee score. They concluded saying even thou literature suggests a trend toward decreased surgical failures with femoral fixation at the joint line with an interference screw, there is no 
difference when postoperative functional outcomes are compared. Future studies are needed with standardized fixation methods and outcomes assessment to determine the importance of femoral fixation ${ }^{[8]}$.

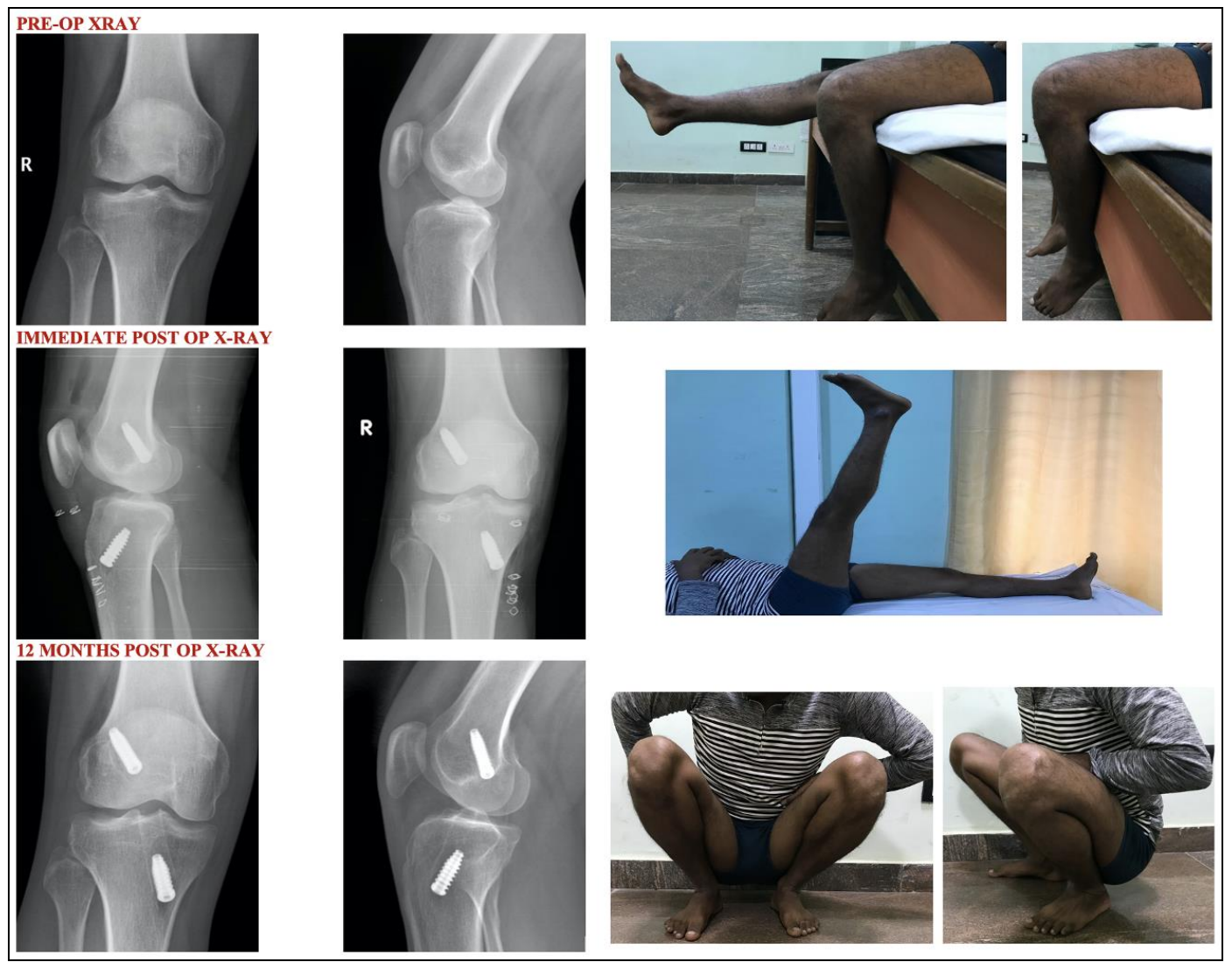

Fig 4: Group 1 Case 1 : Pre and post op Xrays \& 12 months post op clincal pictures of group 1 patient
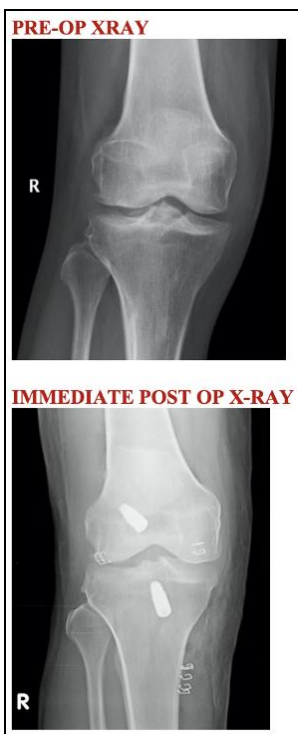

12 MONTHS POST OP X-RAY

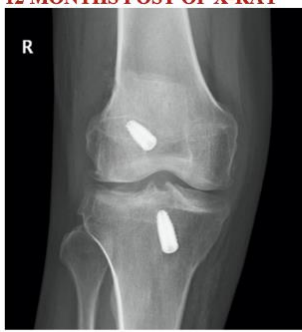

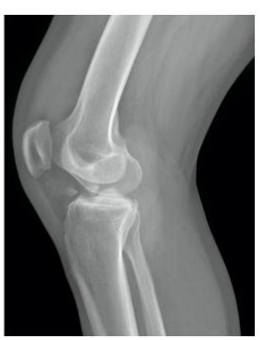
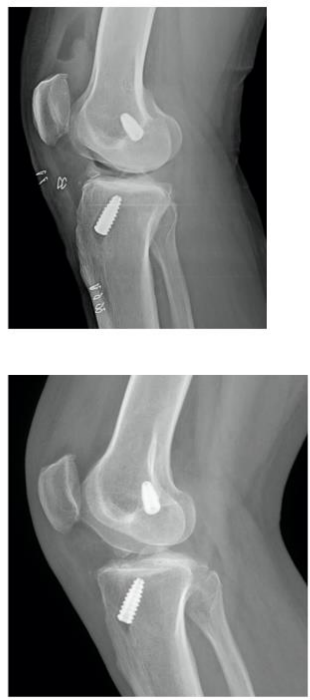
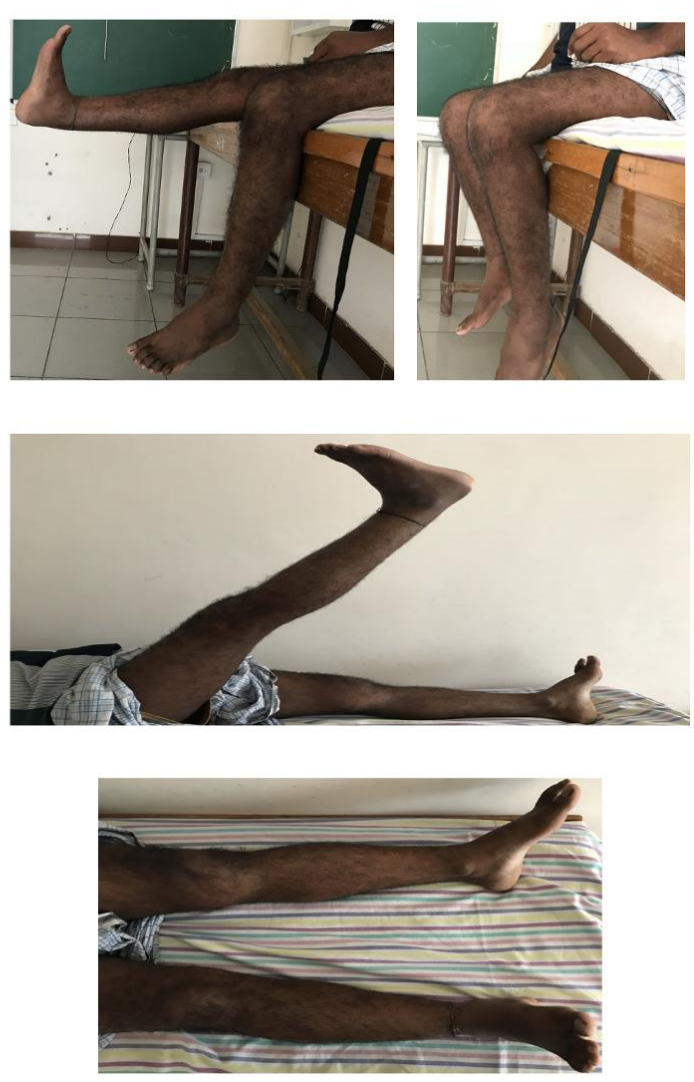

Fig 5: Group 1 Case 2 Pre and post op Xrays \& 12 months post op clincal pictures of group 1 patient 


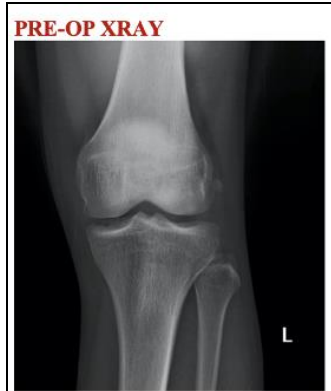

IMMEDIATE POST OP X-RAY

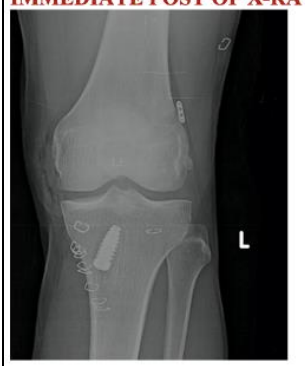

12 MONTHS POST OP X-RAY

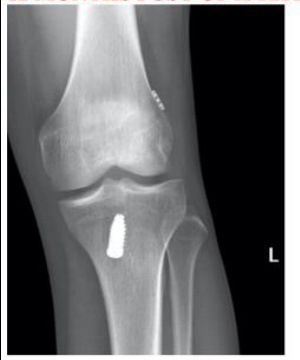

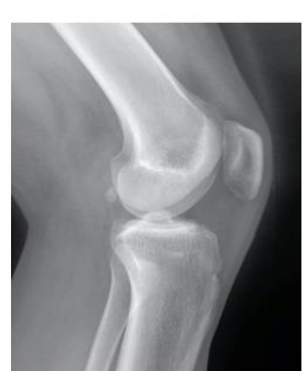
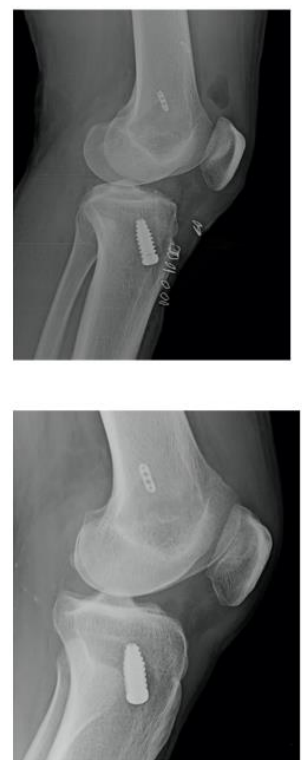
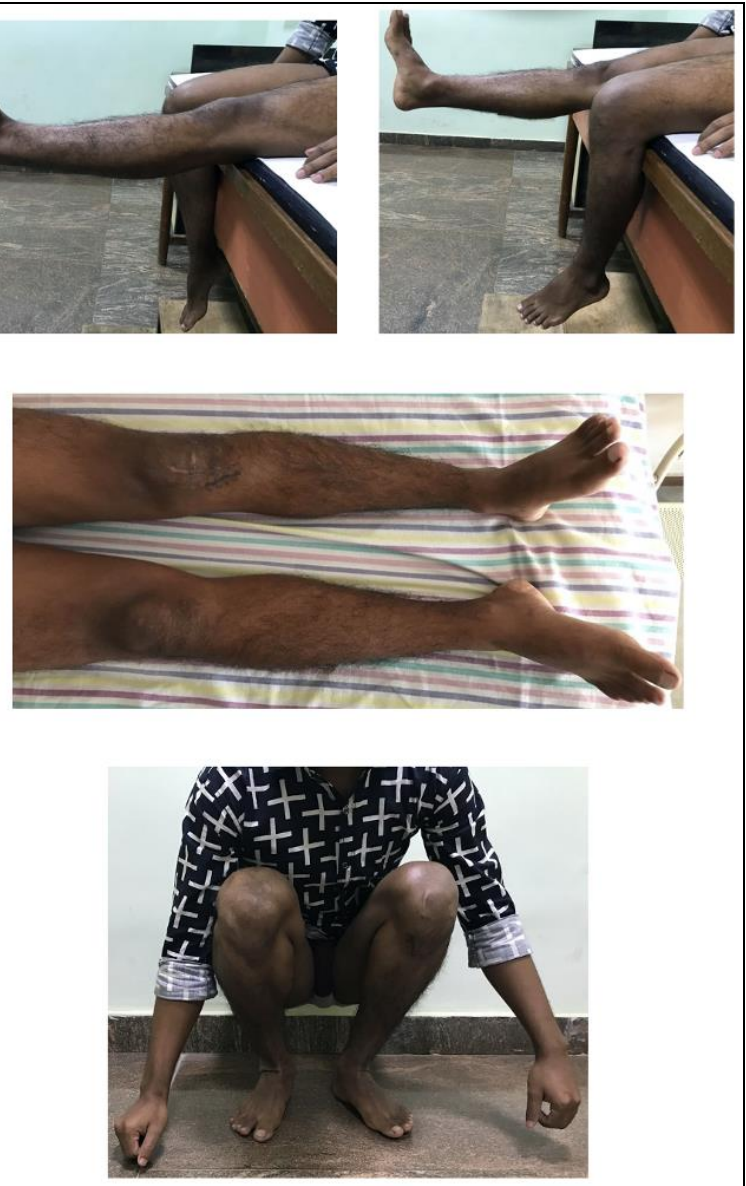

Fig 6: Group 2 Case A: Pre and post op Xrays \& 12 months post op clincal pictures of group 1 patient
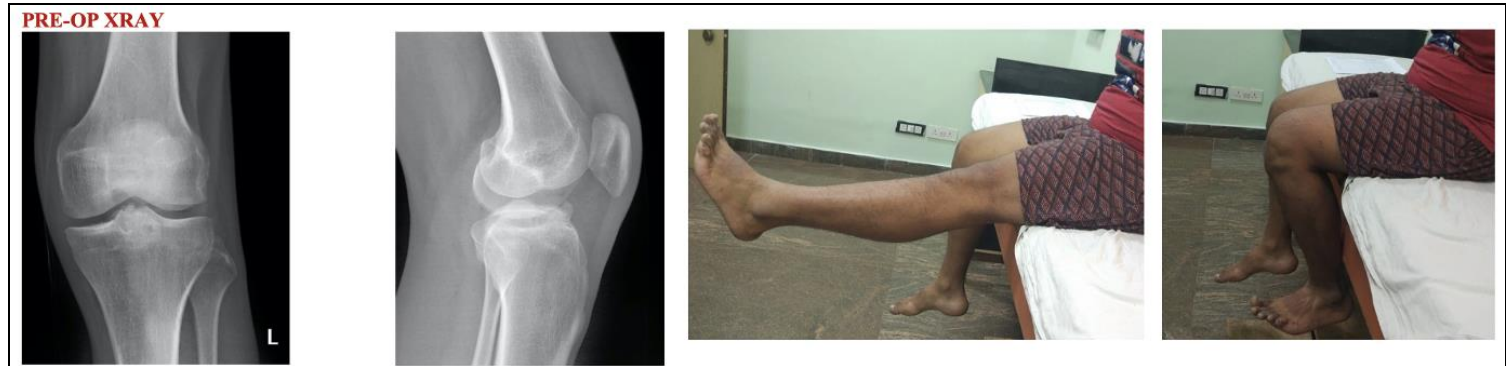

IMMEDIATE POST OP X-RAY
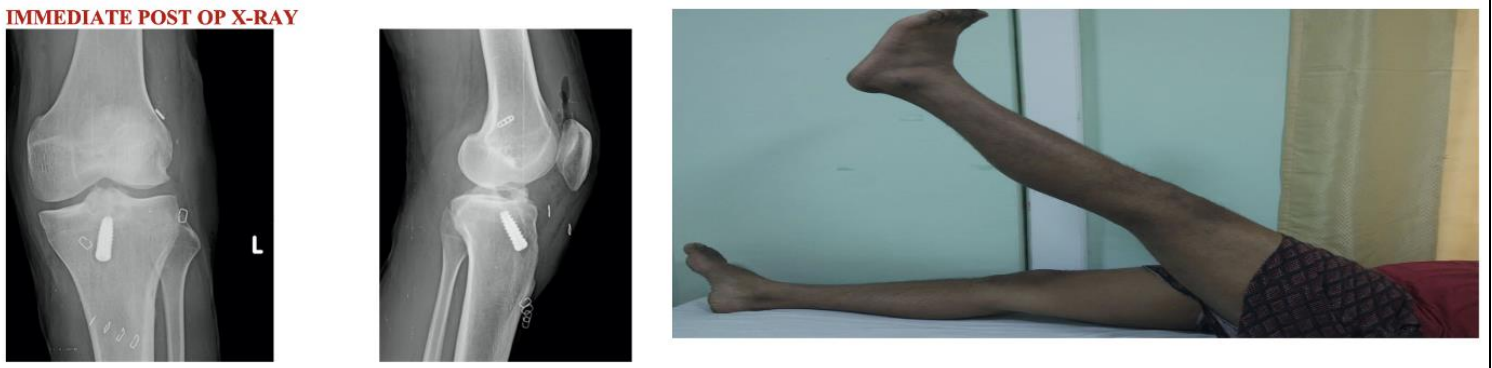

12 MONTHS POST OP X-RAY
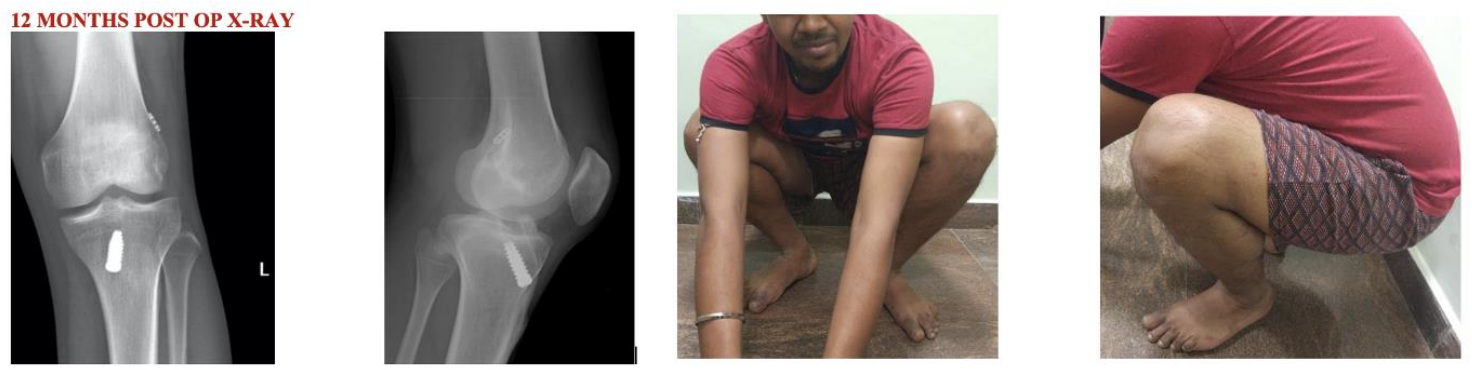

Fig 7: Group 2 Case B: Pre and post op Xrays \& 12 months post op clincal pictures of group 1 patient 


\section{Conclusion}

Arthroscopic anatomic single bundle anterior cruciate ligament reconstruction with quadrupled hamstring graft is an excellent treatment option for anterior cruciate ligament deficient knees. The absence of patellofemoral pain with the use of hamstring graft makes it a more desirable option. The study demonstrated improvement in overall stability of knee with ACL reconstruction using both methods on fixation of the graft. There were fewer complications, and they were comparable between the groups.

The clinical and functional results in our study clarified that both techniques are effective and safe methods for femoral hamstring graft fixation in ACL-reconstruction. However, further studies are needed to confirm these findings during a longer follow up.

Relatively shorter follow up, different operating surgeons and smaller groups are few of the limitations of the study.

\section{References}

1. Azar FM, Beaty JH, Canale ST. Campbell's Operative Orthopaedics. $13^{\text {th }}$ ed. Philadelphia, PA: Elsevier 2017, 2223

2. Browning WM, Kluczynski MA, Curatolo C, Marzo JM. Suspensory versus aperture fixation of a quadrupled hamstring tendon autograft in anterior cruciate ligament reconstruction: A Meta-analysis. Am J Sports Med 2017;45(10):2418-27.

3. Robbe R, Johnson DL. Graft fixation alternatives in Anterior cruciate ligament reconstruction. UPOJ 2002;15:21-7.

4. Hoher J, Livesay GA, Ma CB, Withrow JD, Fu FH, Woo SL. Hamstring graft motion in the femoral bone tunnel when using titanium button/polyester tape fixation. Knee Surg Sports Traumatol Arthrosc. 1999;7:215-219.

5. Scheffler SU, Sudkamp NP, Gockenjan A, Hoffman RF, Weiler A. Biomechanical comparison of hamstring and patel- lar tendon graft anterior cruciate ligament reconstruction techniques: the impact of fixation level and fixation method under cyclic loading. Arthroscopy. 2002;18:304-315.

6. Brand J, Weiler A, Caborn DN, Brown CH Jr, Johnson DL. Graft fixation in cruciate ligament reconstruction. Am J Sports Med 2000;28:761-774.

7. Kim HS, Seon JK, Jo AR. Current trends in Anterior Cruciate Ligament Reconstruction. Knee Surg Relat Res 2013;25(4):165-73.

8. Colvin A, Sharma C, Parides M, Glashow J. What is the best femoral fixation of hamstring autografts in anterior cruciate ligament reconstruction? A meta-analysis. Clin Orthop Relat Res. 2011;469(4):1075-1081.

9. Han DL, Nyland J, Kendzior M, Nawab A, Caborn DN. Intratunnel versus extratunnel fixation of hamstring autograft for anterior cruciate ligament reconstruction. Arthroscopy. 2012;28(10):1555-1566.

10. Ilahi OA, Nolla JM, Ho DM. Intra-tunnel fixation versus extra-tunnel fixation of hamstring anterior cruciate ligament reconstruction: a meta-analysis. J Knee Surg. 2009;22(2):120-129.

11. Ma CB, Francis K, Towers J, Irrgang J, Fu FH, Harner $\mathrm{CH}$. Hamstring anterior cruciate ligament reconstruction: a comparison of bioabsorbable interference screw and endobutton-post fixation. Arthroscopy 2004;20(2):122128.

12. Rose T, Hepp P, Venus J, Stockmar C, Josten C, Lill H. Prospective randomized clinical comparison of femoral transfixation versus bioscrew fixation in hamstring tendon ACL reconstruction: a preliminary report. Knee Surg Sports Traumatol Arthrosc 2006;14(8):730-738.

13. Rozakis M. The role of the RNFA in anterior cruciate ligament graft preparation. AORN J 2014;100(5):500-10.

14. Jadeja H, Yeoh D, Lal M, Mowbray M. Patterns of failure with time of an artificial scaffold class ligament used for reconstruction of the human anterior cruciate ligament. Knee 2007;14(6):439-442.

15. Ekdahl M, Wang JH-C, Ronga M, Fu FH. Graft healing in anterior cruciate ligament reconstruction. Knee Surgery, Sports Traumatology, Arthroscopy. 2008;16(10):935-947.

16. Kyung H-S, Kim S-Y, Oh C-W, Kim S-J. Tendon-tobone tunnel healing in a rabbit model: the effect of periosteum augmentation at the tendon-to-bone interface. Knee Surgery, Sports Traumatology, Arthroscopy. 2003;11(1):9-15.

17. Chen C-H, Liu H-W, Tsai C-L, Yu C-M, Lin I-H, Hsiue G-H. Photoencapsulation of bone morphogenetic protein2 and periosteal progenitor cells improve tendon graft healing in a bone tunnel. American Journal of Sports Medicine 2008;36(3):461-473.

18. Jomha NM, Pinczewski LA, Clingeleffer A, Otto DD. Arthroscopic reconstruction of the anterior cruciate ligament with patellar-tendon autograft and interference screw fixation results at seven years. J Bone Joint Surg Br 1999;81:775-9.

19. Chaudhary D, Monga P, Joshi D, Easwaran J, Bhatia N, Singh A. Arthroscopic reconstruction of the anterior cruciate ligament using bone- patellar tendon-bone autograft. Experience of the first 100 cases. J Orth Surg. 2005;13:147-152.

20. Williams RJ, III Hyman J, Petrigliano F, Rozental T, Wickiewicz TL. Anterior cruciate ligament reconstruction with a four-strand hamstring tendon autograft. J Bone Joint Surg Am 2004;86:225-232.

21. Mahiroğullari M, Kuşkucu M, Kiral A, Pehlivan O, Akmaz I, Tirmik U. Early results of reconstruction of chronic anterior cruciate ligament ruptures using fourstrand hamstring tendon autografts Acta Orthop Traumatol Turc 2005;39:224-230.

22. Kumar PK et al. Functional outcome of arthroscopic reconstruction of anterior cruciate ligament tears. J. Evolution Med. Dent. Sci 2016;5(10):427-432.

23. Gee AO, Kinsella S, Huffman R, Sennett BJ, Tjoumakaris FP. Anterior Cruciate Ligament Reconstruction in Patients Aged > 40 Years: A CaseControl Study. The Physician and Sportsmedicine 2013;41(1):30-34.

24. Wild CY, Steele JR, Munro BJ. Why Do Girls Sustain More Anterior Cruciate Ligament Injuries Than Boys? A Review of the Changes in Estrogen and Musculoskeletal Structure and Function during Puberty. Sports Med. 2012;42(9):733-749.

25. Ferrari JD, Bach BR Jr, Bush-Joseph CA, Wang T, Bojchuk J. Anterior cruciate ligament reconstruction in men and women: An outcome analysis comparing gender. Arthroscopy 2001;17(6):588-96.

26. Chappell JD et al. Effect of fatigue on knee kinetics and kinematics in stop-jump tasks. Am J Sports Med. 2005;33(7):1022-9.

27. Hagino $\mathrm{T}$ et al. Meniscal tears associated with anterior cruciate ligament injury. Arch Orthop Trauma Surg. 2015;135(12):1701-6. 
28. Paschos NK. Anterior cruciate ligament reconstruction and knee osteoarthritis. World J Orthop. 2017;8(3):212217.

29. Shelbourne KD, Patel DV. Timing of surgery in anterior cruciate ligament-injured knees. Knee Surg Sports Traumatol Arthrosc 1995;3(3):148-156.

30. Shelbourne KD, Wilckens JH, Mollabashy A, DeCarlo M. Arthrofibrosis in acute anterior cruciate ligament reconstruction: the effect of timing of reconstruction and rehabilitation. Am J Sports Med. 1991;19(4):332-336.

31. Rousseau $\mathrm{R}$ et al. Complications After Anterior Cruciate Ligament Reconstruction and Their Relation to the Type of Graft: A Prospective Study of 958 Cases. Am J Sports Med 2019;47(11):2543-2549.

32. Hantes ME, Mastrokalos DS, Yu J, Paessler HH. The effect of early motion on tibial tunnel widening after anterior cruciate ligament replacement using hamstring tendon grafts. Arthroscopy 2004;20(6):572-80.

33. Hogervorst T, van der Hart CP, Pels Rijcken TH, Taconis WK. Abnormal bone scans of the tibial tunnel 2 years after patella ligament anterior cruciate ligament reconstruction: correlation with tunnel enlargement and tibial graft length. Knee Surg Sports Traumatol Arthrosc. 2000;8(6):322-38.

34. Wilson TC, Kantaras A, Atay A, Johnson DL. Tunnel enlargement after anterior cruciate ligament surgery. Am J Sports Med 2004;32:543-9.

35. Hoher J, Livesay GA, Ma CB, Withrow JD, Fu FH, Woo SL. Hamstring graft motion in the femoral bone tunnel when using titanium button/polyester tape fixation. Knee Surg Sports Traumatol Arthrosc 1999;7:215-9.

36. Hoher J, Moller HD, Fu FH. Bone tunnel enlargement after anterior cruciate ligament reconstruction: Fact or fiction? Knee Surg Sports Traumatol Arthrosc. 1998;6:231-40. 